\title{
Integrated analysis of the RNA-Seq data of liver hepatocellular carcinoma
}

\author{
H. XUE, L. LUO, Y.T. YAO, L.L. WEI, S.P. DENG, X.L. HUANG* \\ Department of Hepatobiliary Surgery and Cell Transplantation Center, Sichuan Academy of Medical Science and Sichuan Provincial People's \\ Hospital, Chengdu 610071, China
}

*Correspondence: xiaolunhuang@sina.com

Received February 12, 2017/ Accepted June 28, 2017

\begin{abstract}
The present study aimed to explore the genetic changes involved in the liver hepatocellular carcinoma (HCC) development. The RNA-Seq data of 212 HCC tissue samples and 50 normal tissue samples were downloaded using TCGA-Assembler. A total of 4 subgroups were obtained, and 4167, 6279, 5379, and 2548 DEGs were screened in group 1, group 2, group 3, and group 4, respectively. Enrichment analysis found that cell cycle, metabolism, and translation related terms were the most significantly changed functions and pathways. There were 454 genes (1114 pairs), 803 genes (722 pairs), and 788 genes (724 pairs), separately interacted in the condition specific PPI network of group 1, 2, 3, and 4, with MMP2, ATNXN1, F2, and HDAC1 as the hub genes. What's more, using these genes, total 7, 20, 198, and 1 subtype related miRNAs; 35, 50, 47, and 17 subtype related TFs; $1,1,0$, and 2 subtype related drugs were screened in group 1,2, 3, and 4, respectively. The integrated biological analysis on RNA-Seq data provided substantial of bio-molecular related to the HCC development. miR-147b, SP1, and Riboflavin were the subtype-related regulator/drug for HCC. The study about the big data of HCC RNA-Seq data reveals the intrinsic gene expression pattern of the tumor, which provides a novel perspective to understand the heterogeneity of pathogenesis in HCC tumorigenesis.
\end{abstract}

Key words: liver hepatocellular, RNA-Seq, classification, enrichment, miRNA, drug

Among all kinds of primary liver cancers, there are approximately 85-90\% liver hepatocellular carcinoma (HCC) cases [1]. HCC is the $6^{\text {th }}$ most common worldwide cancer, ranked as the most rapidly increasing reason of cancer-related death in the United States [2]. Hepatitis B/C infection, non-alcoholic steatohepatitis, alcoholic liver injury, hereditary metabolic diseases, and environmental carcinogens are the major risk factors for HCC [3]. HCC is usually detected at the time when fewer therapies are available [4], and there is currently no effective cure for it, specifically in the later stage. Because of limited biopsies at the time of HCC diagnosis, biomarkers for patient classification as well as treatments are limited $[5,6]$. Further, majority of HCC diagnoses are now based only on radiological diagnostic criteria $[7,8]$. Therefore, it is urgently needed to get a better understanding of HCC development for better diagnosis and treatments.

High-throughput cDNA sequencing (RNA-Seq) can provide gene expression measurement and has been regarded as an attractive approach capable to alternate microarrays in the analyzing of transcriptome in a comprehensive and unbiased manner [9]. RNA-Seq data are of high reproduc- tion, with few systemic differences among all technical replicates [10]. RNA-Seq has been used as a powerful source in the cancer studies, for instance, Berger et al. [11] applied RNA-seq for melanoma expression profiling, paving a novel avenue for cancer-target discovery. In terms of HCC, Lin et al [12] investigated the key miRNAs in HCC using the small RNA-Seq data, and Huang et al revealed complex transcript patterns in HBV-related HCC samples trough the RNA-Seq data [13]. However, the comprehensive knowledge about the genetic changes in HCC remains to be explored.

To have a more in-depth knowledge of the mechanism of HCC development, Yang et al used the RNA-seq data to identify the critical genes by constructing an interaction network and module analysis [14]. In this study, the RNA-Seq data of 212 HCC tissue samples and 50 normal tissue samples were downloaded. Differentially expressed genes (DEGs) between HCC samples and normal samples were screened and then they were used for the subgroup analysis of all samples. The interacted genes with DEGs in each subgroup were collected for the construction of condition specific protein-protein interaction (PPI) network. The 
regulators (miRNAs and transcript factors) and related drugs of DEGs were also identified, followed by Fisher $t$ test for the subtype related factor selection.

\section{Materials and methods}

Data preprocessing and subgroup classification. All the RNASeqV2 data of HCC were downloaded using TCGAAssembler, including the RPKM (reads per kilobase of exonic sequence per million of total reads sequenced) values of all genes. RPKM is the ratio of reads mapped to genes to reads mapped to genome, and it is a representative value for the expression values of genes. The RPKM values were normalized via the TCC package [15]. Total of 262 samples, containing $212 \mathrm{HCC}$ tissue samples and 50 normal tissue samples were obtained. After the supplementation of the missing value using 1 , the data were conducted $\log 2$ transformation.

NMF [16] was utilized for the subgroup classification of all the cancer samples. Cophenetic correlation coefficient [17] was applied for the optimizing of $\mathrm{k}$ value. The value range of cophenetic correlation coefficient is $0-1$, and the $\mathrm{k}$ value under its highest value is recognized as the ideal $\mathrm{k}$ value.

Screening of DEGs. SAM algorithm [18] was used for the screening of DEGs. To reduce the false positive rate, the genes with adjusted $\mathrm{p}$ value $<0.05$ (Bonferroni adjustment), and $\mid \log 2$ FC (fold change) $\mid<1$ were considered as the DEGs between cancer and control samples. Common DEGs of the 4 subgroups were firstly collected, and the specific DEGs in each subgroup were obtained after removing the common DEGsEnrichment analysis of DEGs. TOPPGENE is an online tool (https://toppgene.cchmc.org/prioritization.jsp) for prioritizing or ranking the candidate genes based on functional similarity to training gene list [19]. Here, to enrich

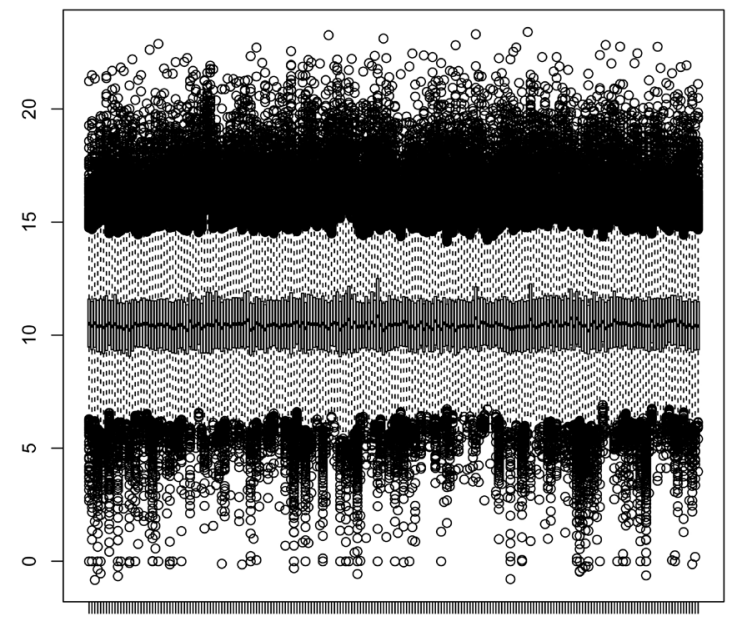

TCGA-CC-5263-01A-01R-A131-07 TCGA-DD-A4NO-01A-11R-A28V-07

Figure 1. Distributions of expression values in the liver hepatocellular RNA-seq samples. The total 212 samples are shown in $\mathrm{x}$ axis, and the transcriptional levels of the whole genome of the samples are shown in y axis. the dysfunctional terms (function and pathway) caused by the DEGs in HCC samples, TOPPGENE was utilized. False discovery rate (FDR) $<0.05$ was set as the threshold.

Interaction network analysis. For the analysis of the interactions between genes (PPI, protein-protein interaction), HPRD (Human Protein Reference Database, http:// hprd.org/) database [6] was retrieved for the gene pairs of the corresponding proteins. Further, the Pearson correlation coefficient of a gene pair under certain condition was calculated based on their expression value, and the pair will be considered as condition specific interacting pair if the coefficient is larger than 0.5 .

cGRNB (Combinatorial Gene Regulation Networks based on user-uploaded gene expression datasets, http://www.scbit. org/cgrnb) database is usually used for studying the complex regulatory relationships, including the miRNA-gene, transcription factor (TF)-gene, and TF-miRNA relationships $[20,21]$. The regulations of miRNA to genes were originally obtained from starBase database, and total 197906 regulatory pairs were collected (669 miRNA and 68646 gens) under the criteria of microRNA readNum $>0$ and biological complexity $>1$. The regulations of TF to genes were obtained based on the USSC sequence information, and total 210637 regulatory pairs were collected (207 TF and 16862 gens).

The interactions between the FDA approved drugs and genes were downloaded from DrugBank (http://www. drugbank.ca/), and total 6108 interaction pairs including 1348 drugs and 1353 target genes were obtained.

Screening of subtype related miRNA/TF/drug. The overlapping significance between DEGs (gene set N) in a subgroup and target genes (gene set Mi) of selected miRNA/ TF/drug (i) were detected using Fisher test [22]. The cut-off threshold was set as $\mathrm{p}<0.05$, and if there is significant overlapping, the corresponding gene associated miRNAs/ TFs/drugs were considered as subtype related. The $\mathrm{p}$ value was computed via the following formula:

$$
\mathrm{p}=\frac{\left(\begin{array}{c}
\mathrm{a}+\mathrm{b} \\
\mathrm{a}
\end{array}\right)\left(\begin{array}{c}
\mathrm{c}+\mathrm{d} \\
\mathrm{c}
\end{array}\right)}{\left(\begin{array}{c}
\mathrm{n} \\
\mathrm{a}+\mathrm{c}
\end{array}\right)}
$$

Whereas "a" means genes are included in both Mi and $\mathrm{N}$, "d" means genes are included in neither Mi nor N, "b" means the genes are only included in $\mathrm{Mi}$, and "c" means the genes are only included in $\mathrm{N}$.

\section{Results}

Data preprocessing and subgroup classification. The distribution of expression values is listed in Figure 1. After the processing, the top 1500 genes with higher variation coefficient were used for the subgroup classification. The cophenetic correlation coefficient was the highest when $\mathrm{k}=4$, 
A
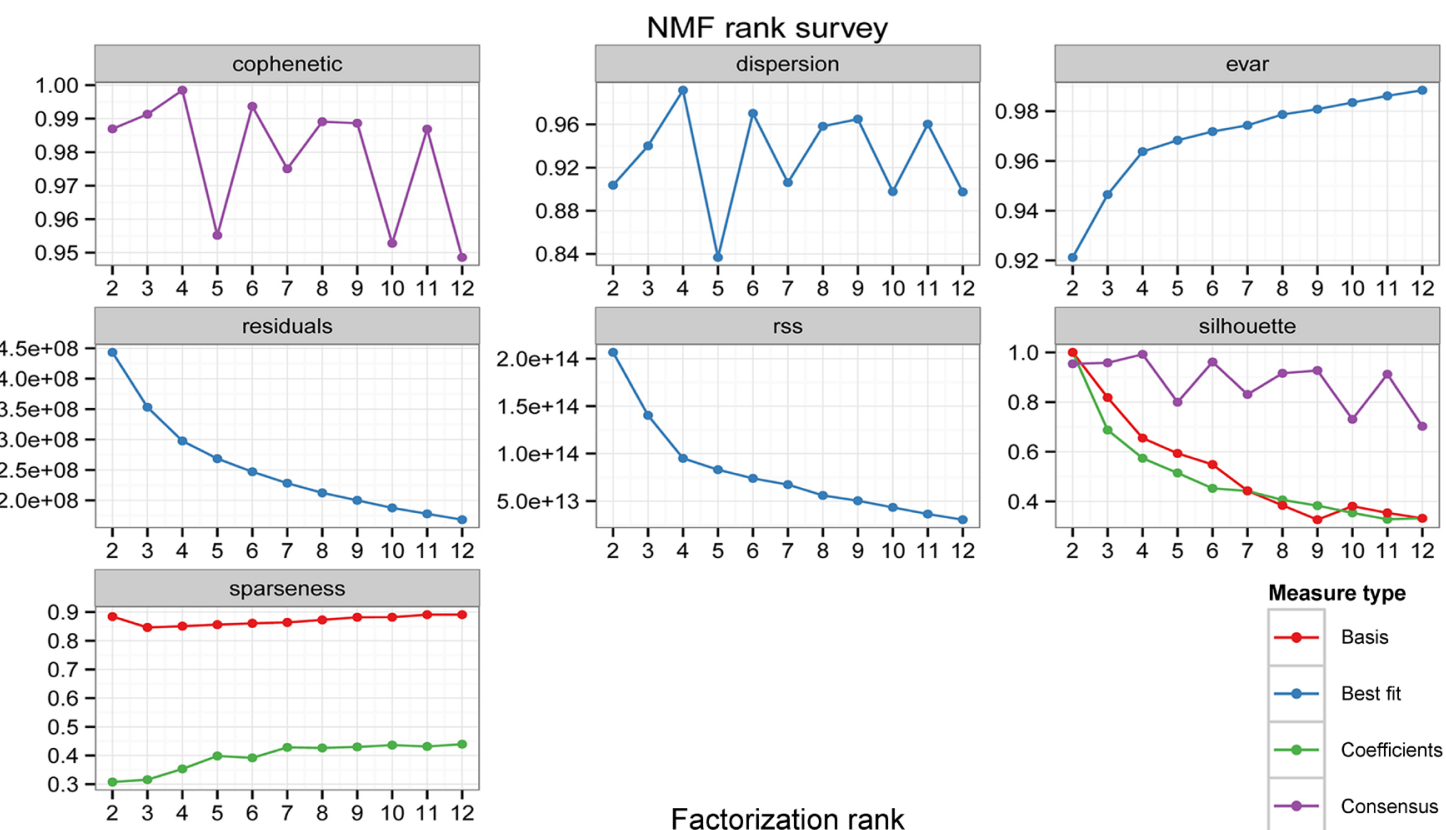

B

Factorization rank

C
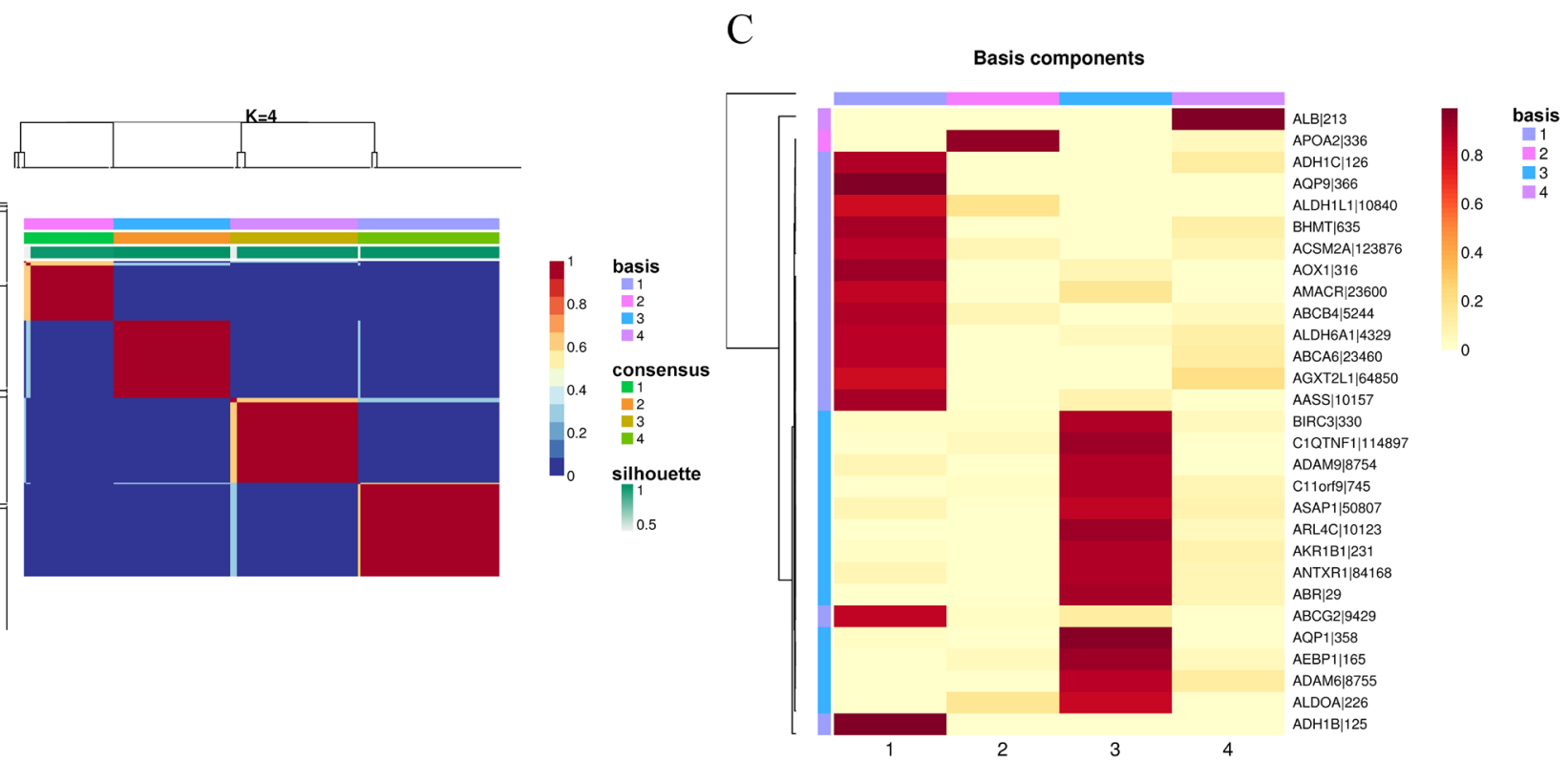

Figure 2. Subgroup classification analyses. A, the evaluation of $k$ value; $B$, the matrixes of the subgroups; $C$, the expression heatmap of key genes in the subgroups.

so total 4 subgroups were screened out: group 1,63 samples; group 2, 40 samples; group 3, 52 samples; and group 4, 57 samples (Figure 2).

DEG screening. SAM algorithm was applied for the screening of DEGs, and in all, the number of DEGs in group 1 , group 2, group 3, and group 4 was 4167, 6279, 5379, and 2548 respectively. The overlapping genes in 2 subgroups or more groups were collected as the final common DEGs sets, comprising 6836 genes (Figure 3). The DEGs were ranked based on their adjusted $\mathrm{p}$ values, and the heatmap of the top 50 DEGs was drawn (Figure 4). The common DEGs were removed from the DEGs in each subgroup to identify the subtype related miRNA/TF/drugs.

Enrichment analysis. Common DEGs and DEGs in the 4 subgroups were subjected to TOPPGENE for the enrichment analysis. Functional enrichment was conducted from there aspects: molecular function, cellular component, and biological progress. 
The most significant enriched terms of the DEGs in the 4 subgroups are shown in Suppl. Table 1. For DEGs in group 1 and 2, similar terms were enriched, and they were cell cycle related functions and metabolism related pathways; for DEGs in group 3, cell cycle functions, acid catabolic related processes, and mRNA processing pathways were enriched; for DEGs in group 4, cell cycle functions, RNA processing related processes, and translation related pathways were enriched.

The function and pathway enrichment result is displayed in Figure 5. The common DEGs were remarkably enriched in transcription and translation related terms. The most significantly enriched cellular component terms included nuclear binding, RNA binding, nucleoplasm, and nucleolus; the most significantly enriched biological progress terms included RNA processing, mRNA processing, RNA splicing, and mRNA metabolic process; the most significantly enriched pathways included spliceosome pathway, ribosome pathway, pyrimidine metabolism pathway, and purine metabolism pathway. No molecular function terms were enriched.

Condition specific protein-protein interaction network. For the DEGs of the 4 subgroups, 11676 interaction pairs were obtained basing on the information from HPRD database. The interacting gene pairs were then screened by calculating their Pearson correlation coefficients, and those with the coefficient larger than 0.5 were considered as the condition specific pairs. The condition specific PPI network of group 1, 2, 3, and 4 was composed of 579 (454 pairs), 1140 genes (1114 pairs), 803 genes (722 pairs), and 788 genes (724 pairs), separately.

Hub genes in the PPI network are usually the disease related genes, thus, the hub genes in the PPI networks of the 4 subgroups were identified by calculating their degrees. The top 10 genes with highest degrees in each subgroup are displayed in Suppl. Table 2. It is observed in the table that the important hub genes in one subgroup were not so important in other groups, such as the hub gene MMP2 in group 1, ATNXN1 in group 2, F2 in groups 3, and HDAC1 in group 4.

Subtype related miRNA/TF/drug. The regulators (miRNA/TF) and related drugs of DEGs in each group were performed by Fisher t test. Total 5, 5, 5, and 1 subtype related miRNAs; 5, 5, 4, and 5 subtype related TFs; 5, 6, 3, and 2 subtype related drugs were screened in group 1, 2, 3, and 4, respectively (Suppl. Table 3). Among all subtype specific factors, miR-30a and SP1 were group 2 specified, while Dactinomycin was group 4 specified.

\section{Discussion}

HCC is a worldwide social and clinical issue, and the biomarkers for its diagnosis and classification remain limited. The RNA-Seq data of the HCC tissue samples and corresponding normal tissue samples were downloaded to explore the underlying mechanism of HCC. The samples were firstly classified into 4 subgroups based on gene expression levels, and 4167, 6279, 5379, and 2548 DEGs were screened in group 1, group 2, group 3, and group 4, respectively. Cell cycle, metabolism, and translation related functions and pathways were the main terms enriched by the DEGs. The condition specific PPI network of group 1, 2, 3, and 4 was composed of 579 genes (454 pairs), 1140 genes (1114 pairs), 803 genes (722 pairs), and 788 genes (724 pairs), separately. MMP2 and HDAC1 was the hub gene the subgroup network, while,

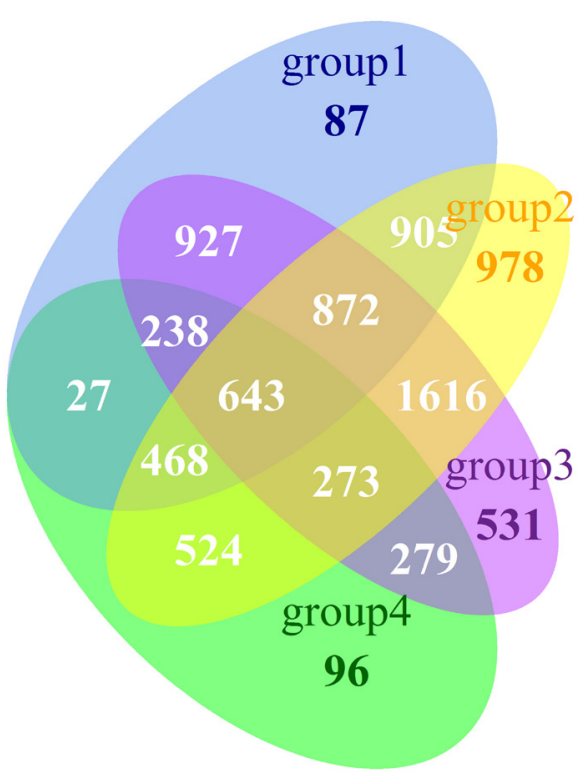

Figure 3. The Venn diagram of differentially expressed genes in the four subgroups.

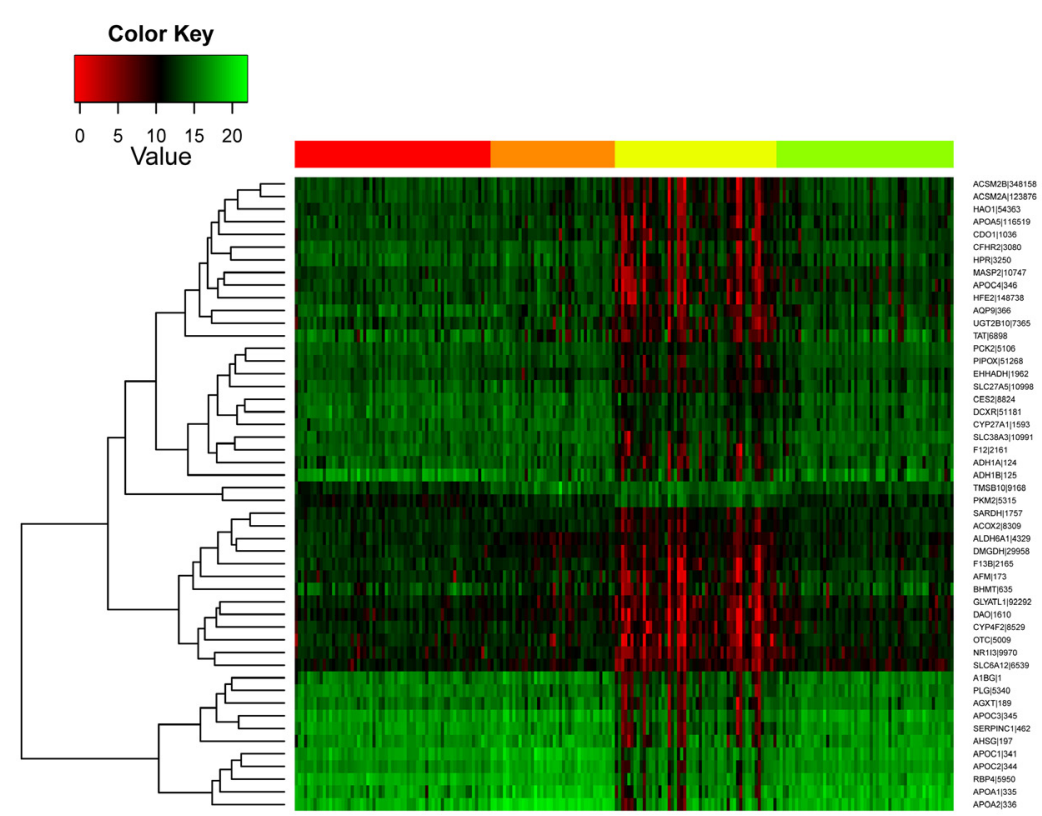

Figure 4. The heatmap of top 50 differentially expressed genes. The 50 genes are shown in $\mathrm{y}$ axis, and samples in four subgroups are shown in $\mathrm{x}$ axis. 


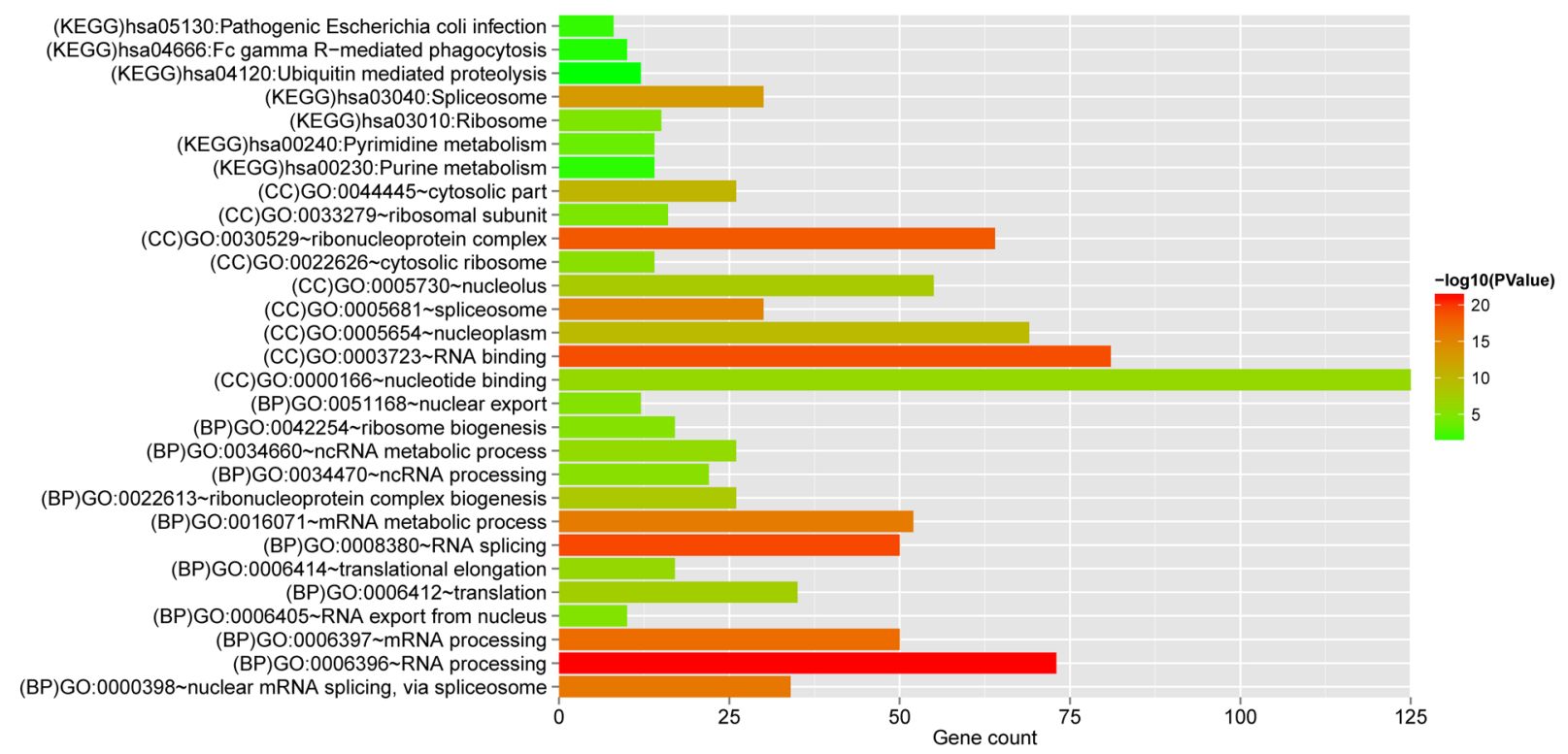

Figure 5. Significantly enriched functional and pathway terms of common DEGs. CC, cellular component; BP, biology process; KEGG, Kyoto Encyclopedia of Genes and Genomes.

miR-147b was found to be the subtype specific regulator, SP1 was the specific TF, and Riboflavin was a subtype-related drug.

MMP (Matrix metalloproteinase) can degrade the components of extracellular matrix. MMP2 is a master regulator in tumor metastasis, and its higher levels are important for the invasions and metastasis of HCC [23]. It is reported that there was a remarkable overexpression in HCC tissues comparing to the adjacent tissues [24], an indicator for the aggressiveness of HCC cells [25]. What's more, the high expression of MMP2 is related to tumor aggressiveness [26], suggesting that samples in group 1 might be highly aggressive HCC. TIMP1, the gene interacted with MMP2 in the PPI network, functions as an endogenous inhibitor of MMP. McKenna et al found that the MMP2:TIMP1 expression increased at the margin tissues than HCC tissues [27]. Therefore, we inferred that the MMP2-TIMP1 variation is a contributor factor for group 1 HCC samples.

HDACs (histone deacetylases) are known to play vital roles in regulating cancer development and progression [28]. The expression of HDAC1 is reported to be overexpressed in HCC, involved in HCC development through systemically regulate the mitotic effectors [29]. Therefore, we inferred that in samples of group 4, cell cycle was significantly changed.

miR-147, induced by different TLR (toll like receptor) agonists, is the murine homologue of human miR-147b [30]. It functions to negatively regulate the LPS-elicited signaling events in the murine macrophages, and its inhibition significantly increased cytokine expression with the stimulation of TLR [31]. TLRs paly various of function in the liver, and it is reported that there is an association between TLR2/9 single nucleotide polymorphisms and the occurrence of HCC [32].
miR-147 has been identified to be differentially expression in recurrent HCC patients comparing with those with no recurrence [33]. As miR-147b was subtype related we inferred that samples in group 3 might be recurrent HCC.

Specificity protein 1 (SP1) is a member of ubiquitously expressed TF family, regulating a larger number of tissue specific genes [34]. The promoter hypomethylation can induce histone hyperacetylation and increase the SP1 binding as well subsequent transactivation, which were associated with poor prognosis of HCC [35]. SP-1 has also been linked to the regulation of invasion and metastasis in liver cancer [36]. Moreover, the HDCA4/Sp1/miR-200a regulatory network is recommended as a potential therapeutic target for the HCC treatment, and the network enhances the proliferation and migration of the cancer cells [37]. Thus, it is assumed that in the group 2 samples, the proliferation and migration might be disrupted.

Riboflavin, playing a vital role in many metabolic reactions, is essential for the normal cellular growth and function [38]. Riboflavin will be converted into flavocoenzymes to realize its biological activity [39]. Riboflavin carrier protein is a growth and development protein, the serum level of which was elevated in HCC [40]. HepG hepatocarcinoma cells have a high demand for riboflavin, and the marginally low riboflavin is shown to induce liver cell stress rapidly [41].

In conclusion, theintegratedbiologicalanalysison RNA-Seq data provided substantial information for the mechanism investigation of HCC. The study about the big data of HCC RNA-Seq data reveals the intrinsic gene expression pattern of the tumor, which provide a novel perspective to understand heterogeneity of pathogenesis in HCC tumorigenesis. 
Supplementary information is available in the online version of the paper.

Acknowledgements: This study was supported by the research grant from Sichuan Provincial Health Department (grant number: 30305030264)

\section{References}

[1] EL-SERAG HB, RUDOLPH KL. Hepatocellular carcinoma: epidemiology and molecular carcinogenesis. Gastroenterology 2007; 132: 2557-2576.

[2] EL-SERAG HB. Hepatocellular carcinoma. N Engl J Med 2011; 365: 1118-1127. doi: 10.1056/NEJMra1001683

[3] CHEN WT, ZHU G, PFAFFENBACH K, KANEL G, STILES $B$ et al. GRP78 as a regulator of liver steatosis and cancer progression mediated by loss of the tumor suppressor PTEN. Oncogene 2014; 33: 4997-5005. doi: 10.1038/onc.2013.437

[4] KASHEFI-KHEYRABADI L, MEHRGARDI MA, WIECHEC E, TURNER AP, TIWARI A. Ultrasensitive detection of human liver hepatocellular carcinoma cells using a label-free aptasensor. Anal Chem 2014; 86: 4956-4960. doi: 10.1021/ ac500375p

[5] TABRIZIAN P, ROAYAIE S, SCHWARTZ ME. Current management of hepatocellular carcinoma. World J Gastroenterol 2014; 20: 10223-10237. doi: 10.3748/wjg.v20.i30.10223

[6] MANCUSO A. Management of hepatocellular carcinoma: Enlightening the gray zones. World J Hepatol 2013; 5: $302-$ 310. doi: $10.4254 /$ wjh.v5.i6.302

[7] KUDO M, IZUMI N, KOKUDO N, MATSUI O, SAKAMOTO $\mathrm{M}$ et al. Management of hepatocellular carcinoma in Japan: Consensus-Based Clinical Practice Guidelines proposed by the Japan Society of Hepatology (JSH) 2010 updated version. Dig Dis. 2011; 29: 339-364. doi: 10.1159/000327577

[8] BRUIX J, SHERMAN M; AMERICAN ASSOCIATION FOR THE STUDY OF LIVER DISEASES. Management of hepatocellular carcinoma: an update. Hepatology 2011; 53: 1020-1022. doi: 10.1002/hep.24199

[9] WANG L, FENG Z, WANG X, WANG X, ZHANG X. DEGseq: an $\mathrm{R}$ package for identifying differentially expressed genes from RNA-seq data. Bioinformatics 2010; 26: 136-138. doi: 10.1093/bioinformatics/btp612

[10] MARIONI JC, MASON CE, MANE SM, STEPHENS M, GILAD Y. RNA-seq: an assessment of technical reproducibility and comparison with gene expression arrays. Genome Res 2008; 18: 1509-1517. doi: 10.1101/gr.079558.108

[11] BERGER MF, LEVIN JZ, VIJAYENDRAN K, SIVACHEN$\mathrm{KO} A, \mathrm{ADICONIS} \mathrm{X}$ et al. Integrative analysis of the melanoma transcriptome. Genome Res 2010; 20: 413-427. doi: $\underline{10.1101 / g r .103697 .109}$

[12] LIN LJ, LIN Y, JIN Y, ZHENG CQ. Investigation of key microRNAs associated with hepatocellular carcinoma using small RNA-seq data. Mol Biol Rep 2014; 41: 4341-4349. doi: 10.1007/s11033-014-3305-4

[13] HUANG Q, LIN B, LIU H, MA X, MO F et al. RNA-Seq analyses generate comprehensive transcriptomic landscape and reveal complex transcript patterns in hepatocellular carcinoma. PLoS One 2011; 6: e26168. doi: 10.1371/journal. pone. 0026168
[14] YANG MR, ZHANG Y, WU XX, CHEN W. Critical genes of hepatocellular carcinoma revealed by network and module analysis of RNA-seq data. Eur Rev Med Pharmacol Sci 2016; 20: 4248-4256.

[15] SUN J, NISHIYAMA T, SHIMIZU K, KADOTA K. TCC: an $\mathrm{R}$ package for comparing tag count data with robust normalization strategies. BMC Bioinformatics 2013; 14: 219. doi: $\underline{10.1186 / 1471-2105-14-219}$

[16] ROSSINI PM, BARKER AT, BERARDELLI A, CARAMIA MD, CARUSO G et al. Non-invasive electrical and magnetic stimulation of the brain, spinal cord and roots: basic principles and procedures for routine clinical application. Report of an IFCN committee. Electroencephalogr Clin Neurophysiol 1994; 91: 79-92.

[17] KURAMAE EE, ROBERT V, ECHAVARRI-ERASUN C, BOEKHOUT T. Cophenetic correlation analysis as a strategy to select phylogenetically informative proteins: an example from the fungal kingdom. BMC Evol Biol 2007; 7: 134.

[18] LARSSON O, WAHLESTEDT C, TIMMONS JA. Considerations when using the significance analysis of microarrays (SAM) algorithm. BMC Bioinformatics 2005; 6: 129.

[19] CHEN J, BARDES EE, ARONOW BJ, JEGGA AG. ToppGene Suite for gene list enrichment analysis and candidate gene prioritization. Nucleic Acids Res 2009; 37: W305-311. doi: $10.1093 / \mathrm{nar} / \mathrm{gkp} 427$

[20] TU K, YU H, HUA YJ, LI YY, LIU L et al. Combinatorial network of primary and secondary microRNA-driven regulatory mechanisms. Nucleic Acids Res 2009; 37: 5969-5980. doi: 10.1093/nar/gkp638

[21] YU H, TU K, WANG YJ, MAO JZ, XIE L et al. Combinatorial network of transcriptional regulation and microRNA regulation in human cancer. BMC Syst Biol 2012; 6: 61. doi: $\underline{10.1186 / 1752-0509-6-61}$

[22] LIAO YY, LEE TS, LIN YM. A Fisher exact test will be more proper. Radiology 2006; 239: 300-301. doi: 10.1148/radiol.2391051114

[23] GAO J, DING F, LIU Q, YAO Y. Knockdown of MACC1 expression suppressed hepatocellular carcinoma cell migration and invasion and inhibited expression of MMP2 and MMP9. Mol Cell Biochem 2013; 376: 21-32. doi: 10.1007/s11010012-1545-y

[24] HUANG D, DU X, YUAN R, CHEN L, LIU T et al. Rock2 promotes the invasion and metastasis of hepatocellular carcinoma by modifying MMP2 ubiquitination and degradation. Biochem Biophys Res Commun 2014; 453: 49-56. doi: 10.1016/j.bbrc.2014.09.061

[25] LIU Q, YANG P, TU K, ZHANG H, ZHENG X et al. TPX2 knockdown suppressed hepatocellular carcinoma cell invasion via inactivating AKT signaling and inhibiting MMP2 and MMP9 expression. Chin J Cancer Res 2014; 26: 410417. doi: 10.3978/j.issn.1000-9604.2014.08.01

[26] MUSSO O, CLÉMENT B, THÉRET N. Assessing matrix metalloproteinase expression and activity in hepatocellular carcinomas. Methods Mol Med 2000; 45: 139-156. doi: 10.1385/1-59259-079-9:13

[27] MCKENNA GJ, CHEN Y, SMITH RM, MENEGHETTI A, ONG C et al. A role for matrix metalloproteinases and tumor host interaction in hepatocellular carcinomas. Am J Surg 2002; 183: 588-594. 
[28] ROPERO S, ESTELLER M. The role of histone deacetylases (HDACs) in human cancer. Mol Oncol 2007; 1: 19-25. doi: 10.1016/j.molonc.2007.01.001

[29] XIE HJ, NOH JH, KIM JK, JUNG KH, EUN JW et al. HDAC1 inactivation induces mitotic defect and caspaseindependent autophagic cell death in liver cancer. PLoS One 2012; 7: e34265. doi: 10.1371/journal.pone.0034265

[30] CHATTERJEE V, BEARD RS JR, REYNOLDS JJ, HAINES $\mathrm{R}$, GUO $\mathrm{M}$ et al. MicroRNA-147b regulates vascular endothelial barrier function by targeting ADAM15 expression. PLoS One 2014; 9: e110286. doi: 10.1371/journal. pone. 0110286

[31] LIU G, FRIGGERI A, YANG Y, PARK YJ, TSURUTA Y et al. miR-147, a microRNA that is induced upon Toll-like receptor stimulation, regulates murine macrophage inflammatory responses. Proc Natl Acad Sci U S A 2009; 106: 15819-15824. doi: 10.1073/pnas.0901216106

[32] JUNJIE X, SONGYAO J, MINMIN S, YANYAN S, BAIYONG $S$ et al. The association between Toll-like receptor 2 single-nucleotide polymorphisms and hepatocellular carcinoma susceptibility. BMC Cancer 2012; 12: 57. doi: 10.1186/1471-2407-12-57

[33] HAN ZB, ZHONG L, TENG MJ, FAN JW, TANG HM et al. Identification of recurrence-related microRNAs in hepatocellular carcinoma following liver transplantation. Mol Oncol 2012; 6: 445-457. doi: 10.1016/j.molonc.2012.04.001

[34] WILSON AJ, CHUEH AC, TÖGEL L, CORNER GA, AHMED N et al. Apoptotic sensitivity of colon cancer cells to histone deacetylase inhibitors is mediated by an Sp1/ Sp3-activated transcriptional program involving immediateearly gene induction. Cancer Res 2010; 70: 609-620. doi: 10.1158/0008-5472.CAN-09-2327
[35] KONG LM, LIAO CG, CHEN L, YANG HS, ZHANG SH et al. Promoter hypomethylation up-regulates CD147 expression through increasing Sp1 binding and associates with poor prognosis in human hepatocellular carcinoma. J Cell Mol Med 2011; 15: 1415-1428. doi: 10.1111/j.15824934.2010.01124.x

[36] PREVIDI S, MALEK A, ALBERTINI V, RIVA C, CAPELLA $\mathrm{C}$ et al. Inhibition of Sp1-dependent transcription and antitumor activity of the new aureolic acid analogues mithramycin SDK and SK in human ovarian cancer xenografts. Gynecol Oncol 2010; 118: 182-188. doi: 10.1016/j.ygyno.2010.03.020

[37] YUAN JH, YANG F, CHEN BF, LU Z, HUO XS et al. The histone deacetylase 4/SP1/microrna-200a regulatory network contributes to aberrant histone acetylation in hepatocellular carcinoma. Hepatology 2011; 54: 2025-2035. doi: 10.1002/hep. 24606

[38] SAID HM, ORTIZ A, MA TY, MCCLOUD E. Riboflavin uptake by the human-derived liver cells Hep G2: mechanism and regulation. J Cell Physiol 1998; 176: 588-594.

[39] MANTHEY KC, CHEW YC, ZEMPLENI J. Riboflavin deficiency impairs oxidative folding and secretion of apolipoprotein B-100 in HepG2 cells, triggering stress response systems. J Nutr 2005; 135: 978-982.

[40] RAO PN, CRIPPIN J, LEVINE E, HUNT J, BALIGA S et al. Elevation of serum riboflavin carrier protein in hepatocellular carcinoma. Hepatol Res 2006; 35: 83-87. doi: 10.1016/j. hepres.2006.03.009

[41] WERNER R, MANTHEY KC, GRIFFIN JB, ZEMPLENI J. HepG2 cells develop signs of riboflavin deficiency within 4 days of culture in riboflavin-deficient medium. J Nutr Biochem 2005; 16: 617-624. 
Suppl. Table 1. Top 5 enriched terms of differentially expressed genes in the four subgroups.

\begin{tabular}{|c|c|c|c|c|}
\hline Category & ID & Name & FDR & Count \\
\hline \multicolumn{5}{|l|}{ Group 1} \\
\hline GO: Molecular Function & GO:0003723 & RNA binding & 8.93E-64 & 611 \\
\hline GO: Molecular Function & GO:0044822 & poly(A) RNA binding & $9.05 \mathrm{E}-57$ & 477 \\
\hline GO: Molecular Function & GO:0003735 & structural constituent of ribosome & $2.47 \mathrm{E}-32$ & 104 \\
\hline GO: Molecular Function & GO:0019899 & enzyme binding & $5.90 \mathrm{E}-14$ & 435 \\
\hline GO: Molecular Function & GO:0032403 & protein complex binding & $1.57 \mathrm{E}-07$ & 270 \\
\hline GO: Cellular Component & GO:0030529 & ribonucleoprotein complex & $2.22 \mathrm{E}-46$ & 294 \\
\hline GO: Cellular Component & GO:0005730 & nucleolus & $1.59 \mathrm{E}-44$ & 612 \\
\hline GO: Cellular Component & GO:0005739 & mitochondrion & $5.48 \mathrm{E}-35$ & 550 \\
\hline GO: Cellular Component & GO:0044391 & ribosomal subunit & $4.12 \mathrm{E}-34$ & 101 \\
\hline GO: Cellular Component & GO:0022626 & cytosolic ribosome & 8.07E-33 & 76 \\
\hline GO: Biological Process & GO:0006614 & SRP-dependent cotranslational protein targeting to membrane & $4.49 \mathrm{E}-41$ & 90 \\
\hline GO: Biological Process & GO:0006613 & cotranslational protein targeting to membrane & $3.81 \mathrm{E}-40$ & 90 \\
\hline GO: Biological Process & GO:0045047 & protein targeting to ER & $3.81 \mathrm{E}-40$ & 92 \\
\hline GO: Biological Process & GO:0072599 & establishment of protein localization to endoplasmic reticulum & $1.18 \mathrm{E}-39$ & 92 \\
\hline GO: Biological Process & GO:0006412 & translation & $1.11 \mathrm{E}-38$ & 250 \\
\hline Pathway & 83036 & Ribosome & $8.42 \mathrm{E}-30$ & 96 \\
\hline Pathway & 477135 & Metabolism & $2.28 \mathrm{E}-18$ & 507 \\
\hline Pathway & 198853 & Cytoplasmic Ribosomal Proteins & $2.28 \mathrm{E}-18$ & 76 \\
\hline Pathway & 106230 & Metabolism of proteins & $5.99 \mathrm{E}-18$ & 261 \\
\hline Pathway & 105965 & Translation & $2.32 \mathrm{E}-17$ & 107 \\
\hline \multicolumn{5}{|l|}{ Group 2} \\
\hline GO: Molecular Function & GO:0003723 & RNA binding & $8.93 \mathrm{E}-64$ & 611 \\
\hline GO: Molecular Function & GO:0044822 & poly(A) RNA binding & $9.05 \mathrm{E}-57$ & 477 \\
\hline GO: Molecular Function & GO:0003735 & structural constituent of ribosome & $2.47 \mathrm{E}-32$ & 104 \\
\hline GO: Molecular Function & GO:0019899 & enzyme binding & $5.90 \mathrm{E}-14$ & 435 \\
\hline GO: Molecular Function & GO:0032403 & protein complex binding & $1.57 \mathrm{E}-07$ & 270 \\
\hline GO: Cellular Component & GO:0030529 & ribonucleoprotein complex & $2.22 \mathrm{E}-46$ & 294 \\
\hline GO: Cellular Component & GO:0005730 & nucleolus & $1.59 \mathrm{E}-44$ & 612 \\
\hline GO: Cellular Component & GO:0005739 & mitochondrion & $5.48 \mathrm{E}-35$ & 550 \\
\hline GO: Cellular Component & GO:0044391 & ribosomal subunit & $4.12 \mathrm{E}-34$ & 101 \\
\hline GO: Cellular Component & GO:0022626 & cytosolic ribosome & 8.07E-33 & 76 \\
\hline GO: Biological Process & GO:0006614 & SRP-dependent cotranslational protein targeting to membrane & $4.49 \mathrm{E}-41$ & 90 \\
\hline GO: Biological Process & GO:0006613 & cotranslational protein targeting to membrane & $3.81 \mathrm{E}-40$ & 90 \\
\hline GO: Biological Process & GO:0045047 & protein targeting to ER & $3.81 \mathrm{E}-40$ & 92 \\
\hline GO: Biological Process & GO:0072599 & establishment of protein localization to endoplasmic reticulum & $1.18 \mathrm{E}-39$ & 92 \\
\hline GO: Biological Process & GO:0006412 & translation & $1.11 \mathrm{E}-38$ & 250 \\
\hline Pathway & 83036 & Ribosome & $8.42 \mathrm{E}-30$ & 96 \\
\hline Pathway & 477135 & Metabolism & $2.28 \mathrm{E}-18$ & 507 \\
\hline Pathway & 198853 & Cytoplasmic Ribosomal Proteins & $2.28 \mathrm{E}-18$ & 76 \\
\hline Pathway & 106230 & Metabolism of proteins & $5.99 \mathrm{E}-18$ & 261 \\
\hline Pathway & 105965 & Translation & $2.32 \mathrm{E}-17$ & 107 \\
\hline \multicolumn{5}{|l|}{ Group3 } \\
\hline GO: Molecular Function & GO:0044822 & poly(A) RNA binding & $6.23 \mathrm{E}-57$ & 562 \\
\hline GO: Molecular Function & GO:0003723 & RNA binding & $3.29 \mathrm{E}-49$ & 685 \\
\hline GO: Molecular Function & GO:0048037 & cofactor binding & $1.36 \mathrm{E}-24$ & 161 \\
\hline GO: Molecular Function & GO:0019899 & enzyme binding & $4.25 \mathrm{E}-22$ & 567 \\
\hline GO: Molecular Function & GO:0050662 & coenzyme binding & $4.86 \mathrm{E}-17$ & 115 \\
\hline GO: Cellular Component & GO:0005730 & nucleolus & $3.89 \mathrm{E}-76$ & 820 \\
\hline GO: Cellular Component & GO:0005654 & nucleoplasm & $4.38 \mathrm{E}-58$ & 663 \\
\hline GO: Cellular Component & GO:0005739 & mitochondrion & $6.04 \mathrm{E}-38$ & 673 \\
\hline GO: Cellular Component & GO:1902494 & catalytic complex & $1.91 \mathrm{E}-28$ & 370 \\
\hline GO: Cellular Component & GO:0044451 & nucleoplasm part & $6.37 \mathrm{E}-28$ & 306 \\
\hline
\end{tabular}




\section{Suppl. Table 1. Contidued}

\begin{tabular}{|c|c|c|c|c|}
\hline Category & ID & Name & FDR & Count \\
\hline GO: Biological Process & GO:0006396 & RNA processing & $2.88 \mathrm{E}-29$ & 338 \\
\hline GO: Biological Process & GO:0016054 & organic acid catabolic process & $2.88 \mathrm{E}-29$ & 143 \\
\hline GO: Biological Process & GO:0046395 & carboxylic acid catabolic process & $2.88 \mathrm{E}-29$ & 143 \\
\hline GO: Biological Process & GO:0044282 & small molecule catabolic process & $2.88 \mathrm{E}-29$ & 172 \\
\hline GO: Biological Process & GO:0019752 & carboxylic acid metabolic process & $8.69 \mathrm{E}-29$ & 437 \\
\hline Pathway & 477135 & Metabolism & $2.16 \mathrm{E}-30$ & 662 \\
\hline Pathway & 160950 & Processing of Capped Intron-Containing Pre-mRNA & $1.03 \mathrm{E}-19$ & 98 \\
\hline Pathway & 198843 & mRNA processing & $1.28 \mathrm{E}-12$ & 84 \\
\hline Pathway & 160977 & Fatty acid, triacylglycerol, and ketone body metabolism & $5.21 \mathrm{E}-12$ & 105 \\
\hline Pathway & 82952 & Valine, leucine and isoleucine degradation & $2.05 \mathrm{E}-11$ & 37 \\
\hline \multicolumn{5}{|l|}{ Group 4} \\
\hline GO: Molecular Function & GO:0003723 & RNA binding & $3.59 \mathrm{E}-82$ & 476 \\
\hline GO: Molecular Function & GO:0044822 & poly(A) RNA binding & $8.23 \mathrm{E}-78$ & 386 \\
\hline GO: Molecular Function & GO:0003735 & structural constituent of ribosome & $1.28 \mathrm{E}-48$ & 100 \\
\hline GO: Molecular Function & GO:0005198 & structural molecule activity & $1.52 \mathrm{E}-08$ & 140 \\
\hline GO: Molecular Function & GO:0000988 & protein binding transcription factor activity & $1.21 \mathrm{E}-05$ & 116 \\
\hline GO: Cellular Component & GO:0030529 & ribonucleoprotein complex & $1.33 \mathrm{E}-69$ & 256 \\
\hline GO: Cellular Component & GO:0005730 & nucleolus & $6.66 \mathrm{E}-67$ & 481 \\
\hline GO: Cellular Component & GO:0044391 & ribosomal subunit & $5.63 \mathrm{E}-52$ & 99 \\
\hline GO: Cellular Component & GO:0005840 & ribosome & $2.25 \mathrm{E}-46$ & 115 \\
\hline GO: Cellular Component & GO:0022626 & cytosolic ribosome & $7.51 \mathrm{E}-42$ & 71 \\
\hline GO: Biological Process & GO:0016071 & mRNA metabolic process & $1.41 \mathrm{E}-68$ & 260 \\
\hline GO: Biological Process & GO:0006396 & RNA processing & $5.20 \mathrm{E}-52$ & 249 \\
\hline GO: Biological Process & GO:0022613 & ribonucleoprotein complex biogenesis & $6.47 \mathrm{E}-49$ & 143 \\
\hline GO: Biological Process & GO:0019080 & viral gene expression & $2.10 \mathrm{E}-48$ & 105 \\
\hline GO: Biological Process & GO:0044033 & multi-organism metabolic process & $3.75 \mathrm{E}-47$ & 107 \\
\hline Pathway & 83036 & Ribosome & $7.90 \mathrm{E}-44$ & 91 \\
\hline Pathway & 105937 & Gene Expression & $7.72 \mathrm{E}-29$ & 304 \\
\hline Pathway & 106230 & Metabolism of proteins & $7.96 \mathrm{E}-29$ & 208 \\
\hline Pathway & 105966 & Eukaryotic Translation Initiation & $3.61 \mathrm{E}-26$ & 83 \\
\hline Pathway & 198853 & Cytoplasmic Ribosomal Proteins & $8.70 \mathrm{E}-26$ & 68 \\
\hline
\end{tabular}

Table 2. Top 10 hub genes in the 4 condition specific protein-protein networks.

\begin{tabular}{|c|c|c|c|c|c|c|c|}
\hline \multicolumn{2}{|c|}{ Group 1} & \multicolumn{2}{|c|}{ Group 2} & \multicolumn{2}{|c|}{ Group 3} & \multicolumn{2}{|c|}{ Group 4} \\
\hline Genes & Degree & Genes & Degree & Genes & Degree & Genes & Degree \\
\hline PCNA & 11 & ATXN1 & 23 & $\mathrm{~F} 2$ & 14 & SP1 & 19 \\
\hline EP300 & 8 & EP300 & 21 & PCNA & 13 & PCNA & 18 \\
\hline SP1 & 8 & HRAS & 14 & PLG & 9 & HDAC1 & 11 \\
\hline MCM3 & 7 & CCDC85B & 14 & SNRPD2 & 8 & NCOA6 & 10 \\
\hline $\mathrm{CDK} 2$ & 7 & TRAF2 & 12 & MCM3 & 8 & MCM3 & 10 \\
\hline MMP2 & 7 & COPS6 & 12 & CSNK2B & 8 & TCERG1 & 9 \\
\hline CD74 & 7 & EEF1G & 12 & CDK2 & 8 & EP300 & 9 \\
\hline SPARC & 6 & PIK3R1 & 11 & SNRPE & 8 & MCM2 & 9 \\
\hline ATN1 & 6 & NAA38 & 11 & ACTB & 8 & MCM7 & 9 \\
\hline MCM2 & 6 & UBE2I & 10 & KNG1 & 7 & SFRS1 & 8 \\
\hline
\end{tabular}

Table 3. Subtype related miRNA/TF/drug in the four subgroups.

\begin{tabular}{|c|c|c|c|}
\hline & miRNAs & TFs & Drugs \\
\hline Group1-specific & $\begin{array}{l}\text { mir-15A, mir-124A, mir-520D, } \\
\text { mir-30A, mir-200B }\end{array}$ & LEF1, FOXO4, E12, MAZ, SP1 & losartan, deferoxamine, collagenase, iron, adenine \\
\hline Group2-specific & $\begin{array}{l}\text { mir-19A, mir-17, mir-200B, } \\
\text { mir-142-3P, mir-30A }\end{array}$ & SP1, ELK1, NRF1, NFY, MYC & $\begin{array}{l}\text { adenosine, glutathione, phosphoric acid, iron, insulin } \\
\text { recombinant, adenosine triphosphate }\end{array}$ \\
\hline Group3-specific & $\begin{array}{l}\text { mir-29A, mir-17-5P, LET-7A, } \\
\text { mir-141, mir-9 }\end{array}$ & ERR1, MAZ, MEIS1, ELK1 & adenosine triphosphate, adenosine, glutathione \\
\hline Group4-specific & mir-506 & MAZ, LEF1, AP4, ELK1, E12 & dactinomycin, phosphoric acid \\
\hline
\end{tabular}

